\title{
Maximizing ecological and evolutionary insight from bisulfite sequencing data sets
}

Amanda J. Lea ${ }^{1^{*}}$, Tauras P. Vilgalys ${ }^{2}$, Paul A.P. Durst ${ }^{3}$, and Jenny Tung ${ }^{1,2,4,5^{*}}$

5 1. Department of Biology, Duke University, Box 90338, Durham, NC 27708, USA

6 2. Department of Evolutionary Anthropology, Box 90383, Durham, NC 27708, USA

7 3. Department of Biology, University of North Carolina at Chapel Hill, CB \#3280, Coker Hall,

8 Chapel Hill, NC 27599

9 4. Institute of Primate Research, National Museums of Kenya, P. O. Box 24481, Karen 00502 ,

10 Nairobi, Kenya

11 5. Duke University Population Research Institute, Box 90420, Durham, NC 27708, USA

12

13 *Corresponding author emails: jt5@duke.edu, amanda.lea@duke.edu

14 Other author emails: tauras.vilgalys@duke.edu, pdurst@unc.edu 


\section{Abstract}

The role of DNA methylation in development, divergence, and the response to

18 environmental stimuli is of substantial interest in ecology and evolutionary biology. Measuring

19 genome-wide DNA methylation is increasingly feasible using sodium bisulfite sequencing. Here,

20 we analyze simulated and published data sets to demonstrate how effect size,

21 kinship/population structure, taxonomic differences, and cell type heterogeneity influence the

22 power to detect differential methylation in bisulfite sequencing data sets. Our results reveal that

23 the effect sizes typical of evolutionary and ecological studies are modest, and will thus require

24 data sets larger than those currently in common use. Additionally, our findings emphasize that

25 statistical approaches that ignore the properties of bisulfite sequencing data (e.g., its count-

26 based nature) or key sources of variance in natural populations (e.g., population structure or cell

27 type heterogeneity) often produce false negatives or false positives, thus leading to incorrect

28 biological conclusions. Finally, we provide recommendations for handling common issues that

29 arise in bisulfite sequencing analyses and a freely available R Shiny application for simulating

30 and performing power analyses on bisulfite sequencing data. This app, available at www.tung-

31 lab.org/protocols-and-software.html, allows users to explore the effects of sequencing depth,

32 sample size, population structure, and expected effect size, tailored to their own system.

34 Keywords: DNA methylation, bisulfite sequencing, cell type heterogeneity, population structure,

35 mixed effects models, ecological epigenetics 


\section{Introduction}

DNA methylation - the covalent addition of methyl groups to cytosine bases - is a gene regulatory mechanism of well-established importance in development, disease, and the response to environmental conditions ${ }^{1-3}$. In addition, shifts in DNA methylation are thought to contribute to the speciation process and the evolution of trait differences between taxa ${ }^{4-6}$, in support of the idea that gene regulation plays a key role in evolutionary change. Because of its contribution to phenotypic diversity, interest in DNA methylation from the ecology and evolutionary biology communities is high ${ }^{7-10}$. This interest has been further encouraged by the development of sodium bisulfite sequencing, a cost-effective approach that allows researchers to measure genome-wide DNA methylation levels at base-pair resolution in essentially any $\operatorname{organism}^{11-13}$.

Such approaches rely on sodium bisulfite treatment of DNA followed by high-throughput sequencing, producing data sets that are collectively termed "bisulfite sequencing data." These data have properties (discussed in the following section) that differ in key ways from other common types of sequencing-based functional genomic data, such as RNA-seq data.

Consequently, several statistical approaches have been developed that are specifically tailored to bisulfite sequencing data sets ${ }^{14-17}$ (Box 1). However, the development, application, and evaluation of these tools has primarily focused on biomedical questions or model systems, with an emphasis on case-control studies and experimental manipulations in a restricted set of species $^{18-20}$. In contrast, ecologists and evolutionary biologists often study non-model organisms, environmental gradients that do not follow a case-control design, and natural

57 populations characterized by complex kin or population structure. They are also typically more

58 limited in their ability to sample pure cell types, and may be interested in effects that are smaller

59 than those reported in the context of major perturbations like cancer or pathogen infection ${ }^{21-23}$.

60 Notably, all of these properties can affect statistical power. 
Our goal in this review is to outline considerations for the analysis of bisulfite sequencing data, tailored specifically to concerns that commonly arise in ecological and evolutionary studies. We first discuss how high-throughput bisulfite sequencing data sets are generated, and how this process leads to several idiosyncrasies that must be taken into account in data analysis. Next, we consider four properties common to ecological and evolutionary data sets that can influence power to detect differential methylation: moderate effect sizes, kinship/population structure, taxonomic differences in DNA methylation patterns, and cell type heterogeneity. We analyze both simulated and published empirical data sets to demonstrate how these four features can affect the power and biological interpretation of differential methylation analysis. Finally, we provide recommendations for handling each issue, with the aim of facilitating robust, well-powered studies of DNA methylation's role in ecological and evolutionary processes.

Common properties of high-throughput bisulfite sequencing data sets sequencing $\left(\mathrm{WGBS}^{13}\right)$ or reduced representation bisulfite sequencing (RRBS ${ }^{11}$ ), rely on the differential sensitivity of methylated versus unmethylated cytosines to the chemical sodium bisulfite (Figure 1). Specifically, treatment of DNA with sodium bisulfite converts unmethylated cytosines to uracil (replicated as thymine after PCR) but leaves methylated cytosines unchanged (in vertebrates, most DNA methylation occurs at cytosines in CG motifs, while, in

81 other taxa, cytosines in $\mathrm{CHG}$ and $\mathrm{CHH}$ are also commonly methylated ${ }^{24,25}$ ). DNA methylation

82 level estimates at a given site can thus be obtained via high-throughput sequencing of bisulfite

83 converted DNA, by comparing the relative count of reads contain a cytosine $(C)$, which reflect

84 an originally methylated DNA base, to the count of reads containing a thymine (T), which reflect

85 an originally unmethylated version of the same base. Current bisulfite sequencing protocols

86 require low amounts of input DNA ${ }^{26,27}$, avoid the use of species-specific array platforms, and 
87 can be applied to organisms without a reference genome ${ }^{28}$, making them an increasingly

88 popular choice for ecologists and evolutionary biologists ${ }^{29}$.

High-throughput bisulfite sequencing data have a number of unique properties that

90 influence both study design and data analysis. First, the raw data are binomially distributed

91 count data, in which both the number of methylated reads (unconverted " $\mathrm{C}$ " bases) and the total

92 read depth (number of methylated "C" bases plus unmethylated "T" bases) at each site contain

93 useful information. For example, a site where 5 of 10 reads are methylated and a site where 50

94 of 100 reads are methylated both have estimated methylation levels of $50 \%$. However,

95 confidence in the methylation level estimate is higher for the second site, where total read depth

96 is much greater. Information about relative confidence can be retained by modeling the raw

97 count data rather than transforming counts to proportions or percentages, and several software

98 packages now implement beta-binomial or binomial mixed effects models that do so ${ }^{14-16,30}$ (Box

99 1). These approaches provide a more powerful alternative to tests that assume continuously

100 varying percentages or proportions (e.g., t-tests, Mann-Whitney U tests, linear models). They

101 also control for count overdispersion, a known property of bisulfite sequencing data that violates

102 the assumptions of commonly used, but false positive-prone ${ }^{30}$, binomial models.

104 of bisulfite sequencing data: often, some samples will have low read depth or missing data at a

105 CpG site where other samples have much higher read depth (especially in RRBS data sets,

106 where read coverage is affected by the sample-specific efficiency and specificity of the

107 restriction enzyme digest: Figure 1, Figure S1). Unlike RNA-seq data sets where read depth

108 variation within a sample captures biological information (i.e., once normalized, lower read

109 counts indicate lower expression levels), variance in read depth across sites in bisulfite

110 sequencing data sets is purely technical and tells us nothing about biological variation in DNA

111 methylation levels at a site. Both read depth and effective sample size will thus vary across sites 
112 in the same data set, and will often do so systematically across different regions of the genome

113 (e.g., near genes or in intergenic regions, which differ in CpG content: Figure S1).

114 Finally, the efficacy of the bisulfite conversion step can vary across samples or groups of

115 samples prepared together, creating global batch effects. Though conversion efficiency is

116 typically high (>98\% of unmethylated cytosines converted to thymine ${ }^{31-34}$ ), small differences in

117 conversion efficiency can have significant effects on genome-wide estimates of DNA

118 methylation levels. In particular, samples with low conversion efficiencies will tend to have

119 systematically upwardly biased estimates of DNA methylation levels relative to samples with

120 higher conversion efficiencies, because fewer unmethylated Cs were converted to Ts. Thus,

121 sample-specific bisulfite conversion rates should be directly estimated and taken into account in

122 downstream analyses (e.g., by including bisulfite conversion rate as a model covariate).

123 Estimates of sample-specific conversion rates can be obtained by spiking in a small amount of

124 unmethylated DNA during library construction (lambda phage DNA is commonly used), mapping

125 the resulting reads to the appropriate genome (e.g., the lambda phage genome), and estimating

126 the proportion of unmethylated cytosines in the spike-in DNA sample that failed to convert ${ }^{31-34}$.

127 For data sets that do not include spike-ins, bisulfite conversion rates can be estimated using the

128 conversion rate of $\mathrm{CHH}$ and $\mathrm{CHG}$ sites in species or cell types in which $\mathrm{CHH}$ and $\mathrm{CHG}$

129 methylation is rare ${ }^{27,35,36}$. However, this approach is less ideal because it cannot differentiate

130 between unmethylated cytosines that failed to convert because of technical reasons, and

131 methylated cytosines that truly occur in a non-CpG context.

133 Effect sizes in ecological and evolutionary studies

134 A primary determinant of power in differential methylation analysis is the distribution of

135 true effect sizes (i.e., the magnitude of the effect of the predictor variable of interest on DNA

136 methylation levels). However, it is not obvious what the distributions of effect sizes for questions

137 of ecological and evolutionary interest are likely to be. While effect size distributions and power 
138 analyses have been published for human disease case-control studies ${ }^{18,20}$, comparable

139 information is not readily available for most other settings. Small or moderate epigenetic

140 changes may still impact gene expression levels and consequently be of interest ${ }^{37-39}$; however,

141 they will require larger sample sizes to detect.

142 To aid researchers in choosing appropriate sample sizes, we estimated effect sizes in

143 data sets from plants, hymenopteran insects, and mammals that address a range of ecological

144 and evolutionary questions, including: (i) developmental and demographic effects (eusocial

145 insect caste differentiation ${ }^{35}$; age ${ }^{31}$ ); (ii) ecological effects (resource availability, including both

146 large differences ${ }^{40}$ and more modest ones ${ }^{31}$ ); (iii) genetic effects (cis-acting methylation

147 quantitative trait $\operatorname{loci}^{41}$ ); and (iv) species differences ${ }^{42,43}$ (Table 1). For comparison, we also

148 include a data set contrasting cancer cells with normal tissue from the same donors ${ }^{21}$, which

149 produces some of the largest effect sizes for differential methylation observed to date.

We first reanalyzed each data set using a uniform analysis pipeline (Supplementary

151 Materials) and estimated two measures of effect size: (i) the mean difference in methylation

152 levels between groups of samples, for binary comparisons (Figure 2A) and (ii) the proportion of

153 variance explained by the variable of interest (Figure S2). This analysis provides an empirical

154 picture of how effect size distributions vary across study types. For example, local genetic

155 variants tend to have large effects on DNA methylation levels, while environmental effects are

156 consistently more modest (Figure 2A; Figure S2). To understand how these differences impact

157 power, we simulated bisulfite sequencing data sets across a range of typical effect sizes and

158 estimated the sample size required to identify differentially methylated sites in each case. All

159 simulations presented in the main text assume that $10 \%$ of the sites in each dataset are true

160 positives, but results from parallel analyses with varying proportions of true positives are shown

161 in Figure S3.

162 Our simulation results suggest that answering many ecological and evolutionary

163 questions will require sample sizes that exceed those used in most current studies (Figure 2B; 
164 Table S1). For example, to identify sites where the predictor variable explains $15 \%$ of the

165 variance in DNA methylation levels (a mean difference between sample groups of $13-14 \%$ in

166 our simulations) with $50 \%$ power requires an estimated 125 samples (250 samples for $80 \%$

167 power and 500 samples for $95 \%$ power). To accommodate the costs of larger sample sizes, we

168 recommend reducing per sample read depth or choosing a reduced representation or capture-

169 based approach rather than WGBS. However, we strongly recommend against pooling DNA

170 samples from multiple individuals into a single library, as this approach reduces power by

171 collapsing the number of biological replicates available for analysis. Global analysis approaches

172 that test for patterns in an entire data set, such as principal components analysis (PCA) or

173 hierarchical clustering, may also be helpful when a data set is too power-limited to compensate

174 for the large multiple hypothesis testing burden incurred in site-by-site analyses. This approach

175 is particularly useful when a predictor variable is associated with small changes in DNA

methylation levels at any given locus, but such changes are common genome-wide.

178 rank in rhesus macaques and caste differences in clonal raider ants ${ }^{32,35}$ ), bisulfite sequencing

179 sample sizes were very small. The macaque study ( $n=3$ high-ranking versus $n=3$ low-ranking

180 animals) did not attempt site-by-site analysis, while the raider ant study ( $n=4$ pools of

181 reproductive phase ants versus $n=4$ pools of brood care phase ants) found no evidence for

182 caste effects on DNA methylation using site-by-site paired t-tests. As shown in Figure 2B (see

183 also Figure S4), this result could have stemmed from low power. In support of this possibility,

184 global analysis separates the sample groups of interest in both data sets. Specifically, the

185 macaque study reported that hierarchical clustering distinguishes between high-ranking $(n=3)$

186 and low-ranking $(n=3)$ individuals, with increased separation when focusing on CpG sites near

187 genes differentially expressed with rank ${ }^{32}$. Similarly, when we re-analyzed the clonal raider ant

188 data using principal components analysis, we found that a principal components analysis of

189 CpG sites cleanly separates reproductive and brood care individuals, particularly along principal 
component 3 (t-test for separation along PC 3: $p=0.022$; Figure $2 \mathrm{C}$ ). Together, these results

191 emphasize the potential utility of global analysis approaches in small studies.

\section{Kinship and population structure}

194 Ecological and evolutionary studies often focus on natural populations that contain

195 related individuals or complex population structure. Accounting for these sources of variance is

196 important because DNA methylation levels are often heritable ${ }^{41,44-46}$. In humans, where genetic

197 effects on DNA methylation have been best studied, genotype-DNA methylation associations

198 have now been reported for tens of thousands of CpG sites ${ }^{33,47-49}$, with average heritability

199 levels of $18 \%-20 \%$ in whole blood ${ }^{45,46}$. As a result, more closely related individuals will tend to

200 exhibit more similar DNA methylation patterns than unrelated individuals. Analyses that do not

201 take genetic relationships into account can therefore produce spurious associations if the

202 predictor of interest also covaries with kinship or ancestry. For example, samples are often

203 collected along transects where climatic variables (e.g., temperature, altitude, rainfall) covary

204 with genetic structure ${ }^{41,50}$. Genetic effects on DNA methylation could thus masquerade as

205 climatic effects if genetic sources of variance are not also modeled.

206 Fortunately, this problem is structurally parallel to problems that have already been

207 addressed in genotype-phenotype association studies, phylogenetic comparative analyses, and

208 research on other functional genomic traits. The most straightforward solution is to use mixed

209 effects models, which can incorporate a matrix of pairwise kinship or shared ancestry estimates

210 to account for genetic similarity (Box 1). Specifically, this matrix is treated as the variance-

211 covariance matrix for the heritable (genetic) component of a random effect variable (the

212 environmental component is usually assumed to be independent across samples, so its

213 variance-covariance is given by the identity matrix). The kinship matrix thus contributes to the

214 predicted value of a heritable response variable, but does not affect the value of nonheritable

215 response variables. Notably, while most approaches for controlling for relatedness implement 
216 linear mixed models that are only appropriate for continuous response variables ${ }^{51-53}$, recently

217 developed binomial mixed models can be used to achieve the same task using count data ${ }^{30}$

218 (Box 1). These approaches avoid the need for transforming bisulfite sequencing data from

219 counts to proportions or ratios, thus preserving information about sequencing depth for each

220 site-sample combination. Additionally, recent tools for calling SNP genotypes directly from

221 bisulfite sequencing reads (e.g., BisSNP ${ }^{54}$ and BS-SNPer ${ }^{55}$ ) can help with constructing

222 kinship/relatedness matrices, although not without error (Box 2).

\section{Taxonomic differences in DNA methylation patterns}

225 Most research on DNA methylation to date has focused on humans and a handful of

226 model systems. However, ecologists and evolutionary biologists study a much broader range of

227 species, and patterns of DNA methylation can vary dramatically among taxa $a^{24,25}$. These

228 differences, too, can impact power and analysis strategies for bisulfite sequencing studies. They

229 also mean that patterns typical of one taxonomic group cannot necessarily be extrapolated to

230 others.

231 To provide some intuition about how the distribution of DNA methylation levels vary

232 across taxa, we synthesized data from published studies of flowering plants, hymenopteran

233 insects, canids, humans, and non-human primates (Table 1). We estimated the mean and

234 variance of DNA methylation level for each CpG site in each data set (Figure 3A-B; Figure S5),

235 and used these values to simulate new data sets for power analyses (Supplementary Materials).

236 We were particularly interested in understanding the impact of variance on power because it is

237 unlikely that a predictor variable of interest will significantly explain variation in DNA methylation

238 levels at a locus where there is little variation to begin with. Importantly, the degree to which

239 genomes are composed of relatively monomorphic (low variance) versus high variance sites

240 systematically varies due to both taxon and sequencing strategy (Figure 3A-B, Figure S5).

241 Our simulations suggest that, all else being equal, power to detect differential 
242 methylation in bisulfite sequencing data is limited by variance. Specifically, for any given sample

243 size with a fixed mean DNA methylation level, power increases as a function of the underlying

244 variance in DNA methylation levels (Figure 3C). These results suggest that analyses of low

245 variance genomes, such as those typical of hymenopteran insects, may require larger sample

246 sizes to detect a given effect than analyses of more variable systems, such as plants or

247 mammals. An alternative, a less expensive approach is simply to filter out low variance sites

248 prior to data analysis. Notably, such filtering will also affect the relative representation of sites in

249 genes, promoters, CpG islands, and other functional compartments of the genome, because

250 some of these compartments are consistently more variable than others (Figure S5).

251 In the current literature, differences in the genome-wide distribution of DNA methylation

252 levels across taxa have led to taxon-biased analysis approaches. For example, in

253 hymenopteran insects (where most of the genome is hypomethylated), several studies ${ }^{35,56,57}$

254 have used a binomial test to classify sites into 'unmethylated' or 'methylated' categories (i.e., all

255 sites that do not pass a given significance threshold are considered 'unmethylated'). Our

256 simulations (Supplementary Materials) suggest that this approach not only loses information

257 about quantitative variation, but is also sensitive to technical aspects of the data, such as

258 sequencing depth. For example, using a binomial test approach, a site with an observed

259 methylation level of $15 \%$ would be considered 'unmethylated' at a read depth of 20x, but

260 'methylated' at a read depth of 26x (Figure S6). This problem likely accounts for the report of

261 high rates of 'sample-specific DNA methylation' (where a site is methylated in one sample, but

262 unmethylated in all other samples) in one recent study ${ }^{35}$. Indeed, our re-analysis of the same

263 data shows that $77 \%$ of putative sample-specific sites can be more parsimoniously explained by

264 greater read depth in the "outlier" sample (Figure S6). Such problems can be readily avoided by

265 not binarizing DNA methylation levels, which are intrinsically continuous traits, and by using

266 count-based models that account for variation in sequencing depth ${ }^{14-16,30}$. 


\section{Cell type heterogeneity}

Epigenetic patterns vary substantially across cell types, contributing to differences in

270 gene expression and biological function among different tissues ${ }^{58}$. In some settings, purified cell

271 types can be isolated (e.g., via fluorescent-activated cell sorting). However, this approach is

272 usually not feasible for biologists working under field conditions or with non-model systems (for

273 which antibodies to cell type-specific markers are often unavailable). Consequently, most

274 ecological and evolutionary studies have generated bisulfite sequencing data from

275 heterogeneous samples, such as whole blood, whole organs, or even whole organisms.

276 Because many variables influence both DNA methylation levels and cell type composition,

277 putatively differentially methylated sites could, in some cases, be more parsimoniously

278 explained by variation in cell type proportions rather than a direct effect of the variable of

279 interest on DNA methylation ${ }^{59}$.

280 If isolating purified cell types is not an option, several alternative approaches can be

281 used to address cell type heterogeneity. The best option is to directly estimate the relative

282 proportion of the primary cell types in each sample using cell staining (e.g., Giemsa or Wright-

283 Giemsa) or flow cytometry techniques. These estimates, or a composite measure of multiple

284 estimates (e.g., the first several principal components of variation in cell type proportions) can

285 be incorporated as covariates in downstream analyses. If no measures of cell type

286 heterogeneity are available for the samples of interest, a second option is to use epigenomic

287 profiles from sorted cells ${ }^{36,60}$ to predict the composition of mixed samples (a process known as

288 'deconvolution'59,61). However, deconvolution estimates may introduce additional error,

289 especially for cell types that occur at low frequency. A third option is to use data from sorted cell

290 populations to understand the degree to which cell type composition could confound the

291 conclusions of a study. If sites that are differentially methylated with respect to the predictor of

292 interest also tend to be differentially methylated by cell type, the analysis may be

293 confounded ${ }^{6,31}$. However, if the between-sample compositional differences that would be 
294 required to produce the observed levels of differential methylation are not biologically plausible,

295 tissue heterogeneity is unlikely to completely explain observed differentially methylated sites ${ }^{6}$.

296 Finally, if data from sorted cell populations are unavailable, researchers can apply

297 methods that account for cell type heterogeneity without the need for reference information ${ }^{62-64}$.

298 However, caution is warranted, as some methods make implicit assumptions that may be

299 violated in a given data set. For example, the program FaST-LMM-EWASher controls for cell

300 type heterogeneity by (i) subsetting the data set to focus on the sites most strongly associated

301 with a predictor variable of interest, and then (ii) calculating pairwise covariance between

302 samples using only these sites. The resulting covariance matrix is included as a proxy for

303 covariance in cell type composition in a mixed effects model ${ }^{62}$. However, FaST-LMM-EWASher

304 makes two important assumptions: that most apparent cases of differential methylation are

305 driven by cell type composition effects, and that true positive associations are therefore both

306 rare and of large effect. These assumptions may hold in some studies, but when violated, this

307 approach can substantially reduce power. For example, an analysis of resource base effects in

308 baboon whole blood identified an association with DNA methylation levels at 1014 sites, after

309 ruling out tissue heterogeneity confounds based on blood smear counts and comparisons

310 against purified cell populations ${ }^{31}$. In comparison, FaST-LMM-EWASher detected a single

311 differentially methylated site in the same data set. Alternative programs that account for cell type

312 heterogeneity while making fewer assumptions (e.g., RefFreeEwas ${ }^{64}$ or SVA ${ }^{63}$ ) may thus be

313 more appropriate. However, we caution that while such approaches can help control for

314 variance due to cell type heterogeneity, none can overcome systematic confounding between

315 cell type composition and a predictor of interest.

\section{Conclusions and tools}

318 Like most other genomic technologies, high-throughput bisulfite sequencing approaches

319 were first optimized in research contexts that afford a high degree of control (e.g., experimental 
case-control studies in model systems) and in systems that boast extensive genomic resources

321 (e.g., humans). However, for ecologists and evolutionary biologists, these approaches often

322 become most exciting when they can be extended to a much more diverse set of species and

323 populations - even if these extensions come with complications. We believe that the biological

324 insights to be gained from studies of DNA methylation in diverse taxa have substantial potential.

325 However, maximizing the yield from these studies will require careful consideration of taxon-

326 specific characteristics, the use of analysis methods appropriate to a data set's structure, and

327 realistic assessments of power. In particular, our results reveal that, with sample sizes that are

328 currently feasible for many ecologists and evolutionary biologists, differential methylation

329 analyses will tend to be moderately or lowly powered. Such studies may still have the potential

330 to reveal interesting and important biology. However, researchers should be aware that they are

331 likely to detect only the largest effect sizes (as is also true for other types of genomic analysis ${ }^{65}$ ),

332 and should consider this bias when drawing biological conclusions.

333 Finally, to help quantify how sample size, effect size, population structure, and modeling

334 approach affect bisulfite sequencing data analysis, we have developed an R Shiny application

335 to perform power analyses like those presented here. This app allows bisulfite sequencing data

336 to be simulated with user-specified properties, is coupled with a set of statistical analysis options

337 to evaluate study power, and outputs the simulated count data for maximal flexibility. The app is

338 freely available at www.tung-lab.org/protocols-and-software.html.

\section{Acknowledgements}

We thank Kasper Hansen and Irene Hernando-Herraez for providing processed file

342 formats from their previously published work. We also thank Noah Snyder-Mackler, Luis

343 Barreiro, and Xiang Zhou for helpful comments and suggestions, Mine Cetinkaya-Rundel for

344 coding suggestions on the R Shiny app, and the Baylor College of Medicine Human Genome

345 Sequencing Center for access to the current version of the baboon genome assembly (Panu 
346 2.0). This work was supported by NIH R21-AG049936 and 1R01GM102562 to JT, NSF BCS-

3471455808 to JT and AJL PAPD is supported by NIH K12GM000678 from the Training,

348 Workforce Development \& Diversity division of the National Institute of General Medical

349 Sciences.

351 Data accessibility

352 Summaries of data availability and accession numbers for previously published data sets

353 are provided in Table S1. Our BisSNP analyses utilized publicly available SNP calls for

354 Arabidopsis accessions downloaded from the 1001 Genomes Project

355 (http://1001genomes.org/data/GMI-MPI/releases/v3.1/). An R Shiny app for simulating bisulfite

356 sequencing data and performing power analysis is available at www.tung-lab.org/protocols-and-

357 software.html.

359 Author contributions

360 AJL and JT conceived the study; AJL, TPV, and PAPD analyzed previously published

361 and simulated data; TPV wrote the R Shiny app; and AJL and JT wrote the manuscript, with

362 input from all co-authors. All authors gave final approval for publication. 
364

365

366

367

368

369

370

371

372

373

374

375

376

377

378

379

380

381

382

383

384

385

386

387

388

389

390

391

392

393

394

395

396

397

398

399

400

401

402

403

404

405

406

407

408

409

410

411

412

413

414

\section{References}

1. Feil, R. \& Fraga, M. F. Epigenetics and the environment: emerging patterns and implications. Nat. Rev. Genet. 13, 97-109 (2011).

2. Jones, P. Functions of DNA methylation: islands, start sites, gene bodies and beyond. Nat. Rev. Genet. 13, 484-92 (2012).

3. Smith, Z. D. \& Meissner, A. DNA methylation: roles in mammalian development. Nat. Rev. Genet. 14, 204-220 (2013).

4. Zhao, Y. et al. Adaptive methylation regulation of $p 53$ pathway in sympatric speciation of blind mole rats, Spalax. Proc. Natl. Acad. Sci. 113, 2146-2151 (2016).

5. Durand, S., Bouché, N., Perez Strand, E., Loudet, O. \& Camilleri, C. Rapid establishment of genetic incompatibility through natural epigenetic variation. Current Biology 22, 326331 (2012).

6. Hernando-Herraez, I. et al. Dynamics of DNA methylation in recent human and great ape evolution. PLoS Genet. 9, e1003763 (2013).

7. Hernando-Herraez, I., Garcia-Perez, R., Sharp, A. J. \& Marques-Bonet, T. DNA Methylation: Insights into Human Evolution. PLoS Genet. 11, 1-12 (2015).

8. Snell-Rood, E. The importance of epigenetics for behavioral ecologists (and vice versa). Behav. Ecol. 19, 2012 (2012).

9. Ledon-Rettig, C. C., Richards, C. L. \& Martin, L. B. Epigenetics for behavioral ecologists. Behav. Ecol. 1-14 (2012). doi:10.1093/beheco/ars145

10. Bossdorf, O., Richards, C. L. \& Pigliucci, M. Epigenetics for ecologists. Ecol. Lett. 11, 106-15 (2008).

11. Gu, H. et al. Preparation of reduced representation bisulfite sequencing libraries for genome-scale DNA methylation profiling. Nat. Protoc. 6, 468-81 (2011).

12. Lister, R., Pelizzola, M., Dowen, R. \& Hawkins, R. Human DNA methylomes at base resolution show widespread epigenomic differences. Nature 462, (2009).

13. Cokus, S. J. et al. Shotgun bisulphite sequencing of the Arabidopsis genome reveals DNA methylation patterning. Nature 452, 215-219 (2008).

14. Dolzhenko, E. \& Smith, A. D. Using beta-binomial regression for high-precision differential methylation analysis in multifactor whole-genome bisulfite sequencing experiments. BMC Bioinformatics 15, 215 (2014).

15. Sun, D. et al. MOABS: model based analysis of bisulfite sequencing data. Genome Biol. 15, R38 (2014).

16. Feng, H., Conneely, K. N. \& Wu, H. A Bayesian hierarchical model to detect differentially methylated loci from single nucleotide resolution sequencing data. Nucleic Acids Res. 42, 1-11 (2014).

17. Hansen, K., Langmead, B. \& Irizarry, R. BSmooth : from whole genome bisulfite sequencing reads to differentially methylated regions. Genome Biol. 13, R83 (2012).

18. Tsai, P. C. \& Bell, J. T. Power and sample size estimation for epigenome-wide association scans to detect differential DNA methylation. Int. J. Epidemiol. 44, 1429-1441 (2015).

19. Ziller, M. J., Hansen, K. D., Meissner, A. \& Aryee, M. J. Coverage recommendations for methylation analysis by whole-genome bisulfite sequencing. Nat. Methods 12, 2-5 (2014).

20. Rakyan, V. K., Down, T. a, Balding, D. J. \& Beck, S. Epigenome-wide association studies for common human diseases. Nat. Rev. Genet. 12, 529-41 (2011).

21. Hansen, K. D. et al. Increased methylation variation in epigenetic domains across cancer types. Nat. Genet. 43, 768-75 (2011).

22. Irizarry, R. et al. The human colon cancer methylome shows similar hypo- and hypermethylation at conserved tissue-specific CpG island shores. Nat. Genet. 41, 178-86 
415

416

417

418

419

420

421

422

423

424

425

426

427

428

429

430

431

432

433

434

435

436

437

438

439

440

441

442

443

444

445

446

447

448

449

450

451

452

453

454

455

456

457

458

459

460

461

462

463

464

465

(2009).

23. Pacis, A. et al. Bacterial Infection Remodels the DNA Methylation Landscape of Human Dendritic Cells. Genome Res. (2015). doi:10.1101/gr.192005.115

24. Feng, S. et al. Conservation and divergence of methylation patterning in plants and animals. Proc. Natl. Acad. Sci. 107, 8689-94 (2010).

25. Zemach, A., McDaniel, I. E., Silva, P. \& Zilberman, D. Genome-wide evolutionary analysis of eukaryotic DNA methylation. Science 328, 916-9 (2010).

26. Adey, A. \& Shendure, J. Ultra-low-input, tagmentation-based whole-genome bisulfite sequencing. Genome Res. 22, 1139-1143 (2012).

27. Boyle, P., Clement, K., Gu, H. \& Smith, Z. Gel-free multiplexed reduced representation bisulfite sequencing for large-scale DNA methylation profiling. Genome Biol. 13, R92 (2012).

28. Klughammer, J. et al. Differential DNA Methylation Analysis without a Reference Genome. Cell Rep. 13, 2621-2633 (2015).

29. Verhoeven, K. J. F., VonHoldt, B. M. \& Sork, V. L. Epigenetics in ecology and evolution: what we know and what we need to know. Mol. Ecol. 25, 1631-1638 (2016).

30. Lea, A., Tung, J. \& Zhou, X. A flexible, efficient binomial mixed model for identifying differential DNA methylation in bisulfite sequencing data. PLoS Genet. 11, e1005650 (2015).

31. Lea, A. J., Altmann, J., Alberts, S. C. \& Tung, J. Resource base influences genome-wide DNA methylation levels in wild baboons ( Papio cynocephalus ). Mol. Ecol. (2016). doi:10.1111/mec.13436

32. Tung, J. et al. Social environment is associated with gene regulatory variation in the rhesus macaque immune system. Proc. Natl. Acad. Sci. 109, 6490-5 (2012).

33. Banovich, N. E. et al. Methylation QTLs are associated with coordinated changes in transcription factor binding, histone modifications, and gene expression levels. PLoS Genet. 10, 1-12 (2014).

34. Becker, C. et al. Spontaneous epigenetic variation in the Arabidopsis thaliana methylome. Nature 480, 245-9 (2011).

35. Libbrecht, R., Oxley, P. R., Keller, L. \& Kronauer, D. J. C. Robust DNA Methylation in the Clonal Raider Ant Brain. Curr. Biol. 26, 1-5 (2016).

36. Kawakatsu, T. et al. Unique cell-type-specific patterns of DNA methylation in the root meristem. Nat. Plants 16058 (2016). doi:10.1038/nplants.2016.58

37. Rönn, T. et al. A six months exercise intervention influences the genome-wide DNA methylation pattern in human adipose tissue. PLoS Genet. 9, e1003572 (2013).

38. Murgatroyd, C. et al. Dynamic DNA methylation programs persistent adverse effects of early-life stress. Nat. Neurosci. 12, 1559-66 (2009).

39. Elliott, E., Ezra-Nevo, G., Regev, L., Neufeld-Cohen, A. \& Chen, A. Resilience to social stress coincides with functional DNA methylation of the CRF gene in adult mice. Nat. Neurosci. 13, 1351-3 (2010).

40. Tobi, E. W. et al. DNA methylation signatures link prenatal famine exposure to growth and metabolism. Nat. Commun. 5, 1-13 (2014).

41. Dubin, M. J. et al. DNA methylation variation in Arabidopsis has a genetic basis and appears to be involved in local adaptation. eLife 4, e05255 (2015).

42. Hernando-Herraez, I. et al. The interplay between DNA methylation and sequence divergence in recent human evolution. Nucleic Acids Res. 43, 8204-8214 (2015).

43. Janowitz Koch, I. et al. The concerted impact of domestication and transposon insertions on methylation patterns between dogs and grey wolves. Mol. Ecol. 25, 1838-1855 (2016).

44. Bell, J. T. et al. DNA methylation patterns associate with genetic and gene expression variation in HapMap cell lines. Genome Biol. 12, R10 (2011). 
466

467

468

469

470

471

472

473

474

475

476

477

478

479

480

481

482

483

484

485

486

487

488

489

490

491

492

493

494

495

496

497

498

499

500

501

502

503

504

505

506

507

508

509

510

511

512

513

514

515

516

45. Bell, J. T. et al. Epigenome-wide scans identify differentially methylated regions for age and age-related phenotypes in a healthy ageing population. PLoS Genet. 8, (2012).

46. McRae, A. F. et al. Contribution of genetic variation to transgenerational inheritance of DNA methylation. Genome Biol. 15, R73 (2014).

47. Shi, J. et al. Characterizing the genetic basis of methylome diversity in histologically normal human lung tissue. Nat. Commun. 5, 3365 (2014).

48. Smith, A. K. et al. Methylation quantitative trait loci (meQTLs) are consistently detected across ancestry, developmental stage, and tissue type. BMC Genomics 15, 145 (2014).

49. Hannon, E. et al. Methylation QTLs in the developing brain and their enrichment in schizophrenia risk loci. Nat. Neurosci. 19, 1-9 (2015).

50. Gugger, P. F., Fitz-Gibbon, S., Pellegrini, M. \& Sork, V. L. Species-wide patterns of DNA methylation variation in Quercus lobata and its association with climate gradients. Mol. Ecol. 25, 1665-1680 (2016).

51. Zhou, X. \& Stephens, M. Genome-wide efficient mixed-model analysis for association studies. Nat. Genet. 44, 821-4 (2012).

52. Kang, H. M. et al. Efficient control of population structure in model organism association mapping. Genetics 178, 1709-23 (2008).

53. $\mathrm{Yu}, \mathrm{J}$. et al. A unified mixed-model method for association mapping that accounts for multiple levels of relatedness. Nat. Genet. 38, 203-8 (2006).

54. Liu, Y., Siegmund, K. D., Laird, P. W. \& Berman, B. P. Bis-SNP: Combined DNA methylation and SNP calling for Bisulfite-seq data. Genome Biol. 13, R61 (2012).

55. Gao, S. et al. BS-SNPer: SNP calling in bisulfite-seq data. Bioinformatics 31, 4006-4008 (2015).

56. Bonasio, R. et al. Genome-wide and caste-specific DNA methylomes of the ants camponotus floridanus and harpegnathos saltator. Curr. Biol. 22, 1755-1764 (2012).

57. Lyko, F. et al. The honey bee epigenomes: Differential methylation of brain DNA in queens and workers. PLoS Biol. 8, (2010).

58. Roadmap Epigenomics Consortium et al. Integrative analysis of 111 reference human epigenomes. Nature 518, 317-330 (2015).

59. Jaffe, A. E. \& Irizarry, R. a. Accounting for cellular heterogeneity is critical in epigenomewide association studies. Genome Biol. 15, R31 (2014).

60. Watkins, N. a et al. A HaemAtlas: characterizing gene expression in differentiated human blood cells. Blood 113, 1-9 (2009).

61. Houseman, E. A. et al. DNA methylation arrays as surrogate measures of cell mixture distribution. BMC Bioinformatics 13, 86 (2012).

62. Zou, J., Lippert, C., Heckerman, D., Aryee, M. \& Listgarten, J. Epigenome-wide association studies without the need for cell-type composition. Nat. Methods 11, 309-11 (2014).

63. Leek, J. T. \& Storey, J. D. Capturing heterogeneity in gene expression studies by surrogate variable analysis. PLoS Genet. 3, e161 (2009).

64. Houseman, E. A., Molitor, J. \& Marsit, C. J. Reference-free cell mixture adjustments in analysis of DNA methylation data. Bioinformatics 30, 1431-1439 (2014).

65. Rockman, M. V. The QTN program and the alleles that matter for evolution: All that's gold does not glitter. Evolution 66, 1-17 (2012).

66. Wu, C., DeWan, A., Hoh, J. \& Wang, Z. A comparison of association methods correcting for population stratification in case-control studies. Ann. Hum. Genet. 75, 418-27 (2011).

67. Lippert, C. et al. FaST linear mixed models for genome-wide association studies. Nat. Methods 8, (2011).

68. Perry, G. et al. Comparative RNA sequencing reveals substantial genetic variation in endangered primates. Genome Res. 22, 602-610 (2012).

69. Piskol, R., Ramaswami, G. \& Li, J. B. Reliable identification of genomic variants from 
bioRxiv preprint doi: https://doi.org/10.1101/091488; this version posted December 4, 2016. The copyright holder for this preprint (which was not certified by peer review) is the author/funder, who has granted bioRxiv a license to display the preprint in perpetuity. It is made available under aCC-BY-ND 4.0 International license.

RNA-seq data. Am. J. Hum. Genet. 93, 641-651 (2013).

70. Horton, M. W. et al. Genome-wide patterns of genetic variation in worldwide Arabidopsis thaliana accessions from the RegMap panel. Nat. Genet. 44, 212-216 (2012).

71. Paradis, E., Claude, J. \& Strimmer, K. APE: Analyses of phylogenetics and evolution in R language. Bioinformatics 20, 289-290 (2004). 


\section{Box 1. Modeling approaches for bisulfite sequencing data}

Binomial regression. A binomial distribution intuitively describes bisulfite sequencing data generated for a given sample, $i$, at a given site: the number of methylated counts $(m)$ represents the number of 'successes' in an experiment with $t$ trials and $p$ probability of success. Here, $t$ translates to the total read depth and $p$ to the (unobserved) true methylation level.

$$
m_{i} \sim \operatorname{Bin}\left(t_{i}, p_{i}\right)
$$

However, bisulfite sequencing data are overdispersed (i.e., show greater variance than expected) relative to binomial expectations. Thus, using a binomial regression to model bisulfite sequence data can result in an extremely high rate of false positives and is not recommended $^{14,30}$.

Beta binomial regression. To account for overdispersion, beta binomial regressions have been proposed for bisulfite sequencing data ${ }^{14-16}$. Here, the parameter $p_{i}$ from the binomial setting (equation 1 ) is itself treated as a random variable that follows a two-parameter beta distribution.

$$
\begin{gathered}
p_{i} \sim \operatorname{Beta}\left(\alpha_{i}, \beta_{i}\right) \text { where } \alpha_{i} \geq 0 \text { and } \beta_{i} \geq 0 \\
m_{i} \sim \operatorname{Bin}\left(t_{i}, p_{i}\right)
\end{gathered}
$$

The beta distribution is then re-parameterized as a beta binomial with parameters $t_{i}, \pi_{i}$ (equal to $\left.\alpha_{i} /\left(\alpha_{i},+\beta_{i}\right)\right)$, and $\gamma$ to capture overdispersion.

$$
\begin{gathered}
m_{i} \sim \operatorname{BetaBinomial}\left(t_{i}, \pi_{i}, \gamma\right) \\
\log \left(\frac{\pi_{i}}{1-\pi_{i}}\right)=\beta_{0}+x_{i} \beta_{x}
\end{gathered}
$$

Here, $\pi_{i}$ is the analog of the binomial probability of success $\left(p_{i}\right)$ and can be interpreted as the underlying true methylation level (note that the binomial distribution is a special case of the beta binomial distribution when $\gamma=0$ ). $\pi_{i}$ is passed through a logit link function in order to transform probability values (which are bounded between 0 and 1 ) to a continuous space for linear modeling. Transformed values are modeled as a function of an intercept $\left(\beta_{0}\right)$, the predictor variable of interest $\left(x_{i}\right)$, and its effect size $\left(\beta_{x}\right)$.

Linear mixed effects models. While beta-binomial regressions have become a popular tool for modeling bisulfite sequencing data, these models are not appropriate for data sets that contain related individuals or population structure. Such data sets require approaches that can account for genetic covariance (i.e., nonindependence) among samples, such as linear mixed effects models.

$$
\begin{gathered}
\boldsymbol{y}=\beta_{0}+\boldsymbol{x} \beta_{j}+\boldsymbol{g}+\boldsymbol{\varepsilon} \\
\boldsymbol{\varepsilon} \sim M V N_{n}\left(0, \sigma_{e}{ }^{2} \boldsymbol{I}\right) \\
\boldsymbol{g} \sim M V N_{n}\left(0, \sigma_{g}^{2} \boldsymbol{K}\right) \\
\sigma_{e}{ }^{2}=\sigma^{2}\left(1-h^{2}\right) \text { and } \sigma_{g}{ }^{2}=\sigma^{2} h^{2}
\end{gathered}
$$

Here, $\boldsymbol{y}$ is a vector of continuously distributed methylation levels (obtained by normalizing $m / t$ ) and $\boldsymbol{g}$ is a vector of random effects with a covariance structure determined by the genetic relatedness among individuals in the sample (described by $K$, a user-defined $\mathrm{n} \times \mathrm{n}$ pairwise relatedness matrix) and the heritability of the DNA methylation trait $\left(h^{2}\right.$, which can be decomposed into its genetic and environmental components). $I$ is an $\mathrm{n} \times \mathrm{n}$ identity matrix. 


$$
\begin{gathered}
m_{i} \sim \operatorname{Bin}\left(t_{i}, p_{i}\right) \\
\log \left(\frac{\boldsymbol{p}}{1-\boldsymbol{p}}\right)=\beta_{0}+\boldsymbol{x} \beta_{j}+\boldsymbol{g}+\boldsymbol{\varepsilon}
\end{gathered}
$$

Where $\boldsymbol{\varepsilon}, \boldsymbol{g},{\sigma_{e}}^{2}$, and ${\sigma_{g}}^{2}$ are described as in eq. (4)

\section{This model essentially combines the linear mixed model with the beta binomial regression. The} variable $\boldsymbol{p}$ now reflects the vector of true methylation levels for all samples and is passed through a logit link function for linear modeling. The genetic covariance, as well as the overdispersion, is captured by the random effects component.

\section{Summary of model properties}

\begin{tabular}{|c|c|c|c|}
\hline Method & $\begin{array}{c}\text { Models the count- } \\
\text { based nature of the } \\
\text { data }\end{array}$ & $\begin{array}{l}\text { Models genetic } \\
\text { covariance }\end{array}$ & $\begin{array}{l}\text { Programs that } \\
\text { implement the method }\end{array}$ \\
\hline Binomial regression & Yes* & No & $\mathrm{R}$ and many others \\
\hline $\begin{array}{l}\text { Beta-binomial } \\
\text { regression }\end{array}$ & Yes & No & $\begin{array}{c}\mathrm{DSS}^{16}, \mathrm{MOABS}^{15} \\
\text { RadMeth }^{14}\end{array}$ \\
\hline $\begin{array}{l}\text { Linear mixed effects } \\
\text { model }\end{array}$ & No & Yes & $\begin{array}{c}\text { GEMMA }^{51}, \text { EMMA }^{52} \\
\text { EMMAX }^{66}, \text { FaST-LMM }\end{array}$ \\
\hline $\begin{array}{l}\text { Binomial mixed } \\
\text { effects model }\end{array}$ & Yes & Yes & $\mathrm{MACAU}^{30}$ \\
\hline
\end{tabular}

*Binomial regression is never recommended. Because bisulfite sequencing data are overdispersed relative to the assumptions of this model, binomial regression analyses tend to generate many false positives. 
582

583

584

585

586

587

588

589

590

591

592

593

594

595

596

597

598

599

600

601

602

603

604

605

606

607

608

609

610

611

612

613

614

615

616

617

618

619

620

621

\section{Box 2. Calling genotypes from bisulfite sequencing data}

Like other high-throughput sequencing assays ${ }^{68,69}$, bisulfite sequencing studies generate sequencing reads that contain information about genetic variation. Calling variants or genotypes from these data may be of interest for detecting genetic effects on DNA methylation levels (i.e., methylation quantitative trait loci, or meQTL), verifying sample identity, or controlling for genetic relatedness in downstream analyses. However, typical SNP-calling algorithms are not well suited to bisulfite sequencing data because the $C$ to $T$ conversion obscures true $C / T$ polymorphisms. Several recently developed software packages attempt to overcome these challenges ${ }^{54,55}$. To assess the performance of one such program, BisSNP ${ }^{54}$, we analyzed a whole genome bisulfite sequencing data set for 29 Arabidopsis thaliana accessions ${ }^{41}$ where SNP calls were also available from whole genome sequencing through the 1001 Genomes Project and, for a subset of these individuals $(n=25)$, genotype array data ${ }^{70}$.

Using BisSNP under default recommendations (Supplementary Materials), we identified 235,338 biallelic variable sites. This set was highly skewed to transitions ( $n=234,512$ transitions, $99.65 \%$ of all called sites). Only $45 \%(n=106,925)$ of variants called using BisSNP represent putatively 'true' variants that were also identified in the 1001 Genomes resequencing data, but transversions were much more likely to be 'true' variants than transitions $(90.3 \%$ compared to $45.3 \%$ ). More stringent variant call filtering (variant quality $\geq 50$ rather than $\geq 30$ ) increased the proportion of likely true variants to $50.3 \%$, but at the cost of retaining only $4.7 \%$ of the original sites. However, for previously identified variants in the BisSNP call set, BisSNP genotype calls and genotype array data agreed $87.5 \%$ of the time, with transversions agreeing more often than transitions (93.1\% compared to $87.4 \%$ ). Thus, BisSNP appears to provide relatively high-quality genotyping information for known variants.

However, our analyses do suggest that BisSNP genotypes provide a reliable way to verify sample identity and capture population structure. Using the set of biallelic SNPs that were identified by BisSNP, the 1001 Genomes Project, and the array data $(n=3,553$ SNPs overlapped between all 3 methods for $n=25$ accessions), a neighbor joining tree ${ }^{71}$ clearly clusters samples by accession. The single exception was a WGBS sample that may be mislabeled, as the BisSNP calls clustered separately from the resequencing and array genotype calls for this accession. Further, the pairwise genetic covariance matrix generated from BisSNP calls was highly consistent with the genetic covariance matrix generated from whole genome resequencing data (Mantel test $r=0.873, p<10^{-6}$ ). Perhaps more importantly, the differences we did detect had marginal effects on differential DNA methylation analysis. Specifically, when we analyzed possible methylation quantitative trait loci (meQTL) in the Arabidopsis data set (Supplementary Materials), meQTL effect sizes were highly consistent between analyses using BisSNP calls to estimate population structure and analyses using whole genome resequencing data (Spearman's rho=0.925, $\mathrm{p}<10^{-15}$ ). 
bioRxiv preprint doi: https://doi.org/10.1101/091488; this version posted December 4, 2016. The copyright holder for this preprint (which was not certified by peer review) is the author/funder, who has granted bioRxiv a license to display the preprint in perpetuity. It is made available under aCC-BY-ND 4.0 International license.

622 Table 1. RRBS and WGBS data sets reanalyzed in this study

623

\begin{tabular}{|c|c|c|c|c|}
\hline Species & $\begin{array}{c}\text { Predictor of } \\
\text { interest }\end{array}$ & Contrast & Method & Citation \\
\hline $\begin{array}{l}\text { Dog (Canis lupus familiaris) } \\
\text { and wolf (Canis lupus) }\end{array}$ & Species differences & dog versus wolf & RRBS & 43 \\
\hline Human (Homo sapiens) & $\begin{array}{l}\text { Gestational famine } \\
\text { during the Dutch } \\
\text { Hunger Winter }\end{array}$ & $\begin{array}{c}\text { famine-exposed versus same } \\
\text { sex unexposed sibling }\end{array}$ & RRBS & 40 \\
\hline Yellow baboon (Papio cynocephalus) & Age & continuous age values & RRBS & 31 \\
\hline Yellow baboon & Resource base & $\begin{array}{l}\text { wild-feeding versus human } \\
\text { refuse-supplemented }\end{array}$ & RRBS & 31 \\
\hline Clonal raider ant (Cerapachys biroi) & Caste & $\begin{array}{l}\text { reproductive phase versus } \\
\text { brood care phase }\end{array}$ & WGBS & 35 \\
\hline Human & Cancer status & $\begin{array}{l}\text { normal versus colorectal } \\
\text { tumor samples (paired) }\end{array}$ & WGBS & 21 \\
\hline $\begin{array}{c}\text { Human, orangutan (Pongo abelii), } \\
\text { gorilla (Gorilla gorilla), and chimpanzee } \\
\text { (Pan troglodytes) }\end{array}$ & Species differences & $\begin{array}{c}\text { human versus other great } \\
\text { apes }\end{array}$ & WGBS & 42 \\
\hline Mouseear cress (Arabidopsis thaliana) & $\begin{array}{l}\text { Local genetic } \\
\text { variation }\end{array}$ & $\begin{array}{c}\text { nearby (putatively cis-acting) } \\
\text { genotype }\end{array}$ & WGBS & 41 \\
\hline
\end{tabular}

\section{4}




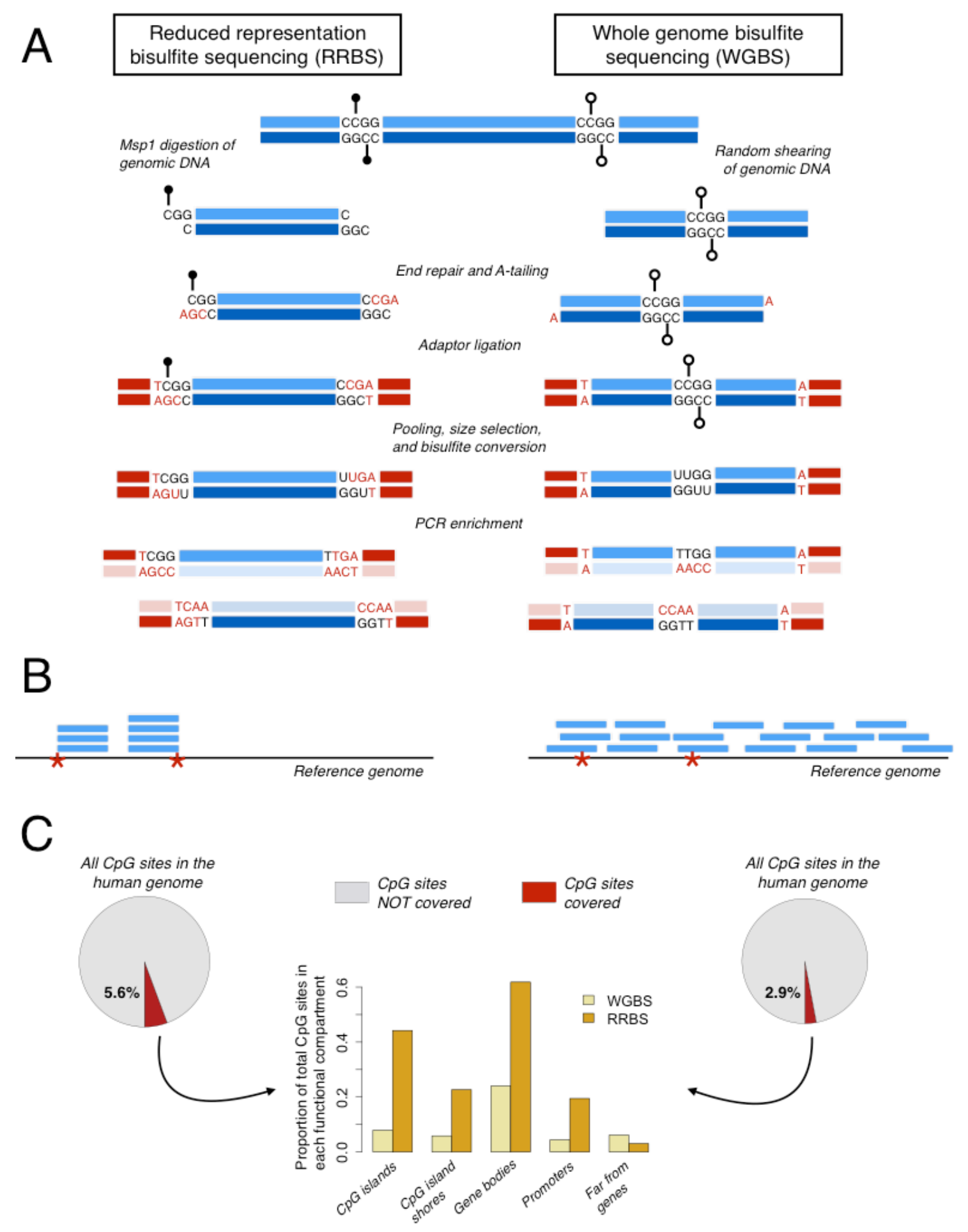

Figure 1. Overview of reduced representation bisulfite sequencing (RRBS; left side of figure) and whole genome bisulfite sequencing (WGBS; right side of figure). (A) Steps required to prepare an RRBS or WGBS library from genomic DNA. Methylated CpG sites are denoted with black lollipops and unmethylated $\mathrm{CpG}$ sites are denoted with open lollipops. Bases artificially 631 introduced during library preparation due to end repair or A-tailing are colored red. Fragments 
632 depicted in the RRBS library prep start and end with the Msp1 digest sites (CCGG) flanking the initial piece of genomic DNA. (B) Read pileups after mapping RRBS and WGBS libraries to a reference genome (red asterisks mark Msp1 digestion sites). In contrast to data from WGBS, reads from RRBS libraries cover a small fraction of the genome. Further, because genomic DNA is fragmented with Msp1 and then size selected (usually for fragments $150-300 \mathrm{bp}$ in length), all fragments retained during the library prep should start and end with an Msp1 recognition site and the pool of fragment will be enriched for $\mathrm{CpG}$ sites. Sequencing reads that are shorter than the original fragment length will localize to the Msp1 recognition site associated with either the 5' or 3' end of the original fragment. (C) Pie charts showing the fraction of all CpG sites in the human genome covered by an RRBS experiment versus a WGBS experiment with similar total read depths (20 million reads generated in silico). Bar charts show, for each method, the proportion of measured CpG sites that fall in gene bodies (between the TSS and the TES), promoters (2 kb upstream of the TSS), CpG islands, and regions far from genes 645 (>100 kb from any annotated TSS or TES). 
A

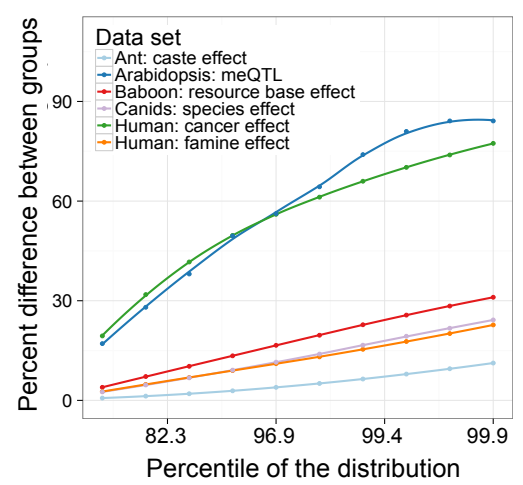

B

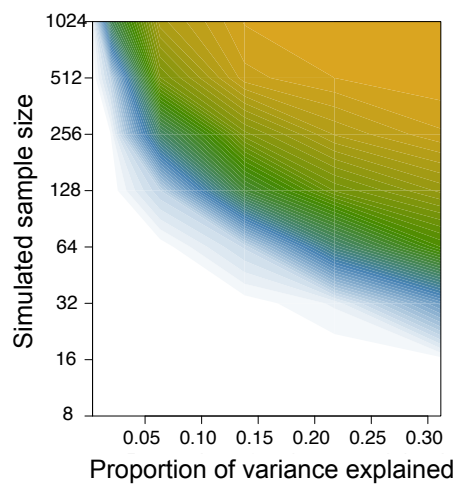

C

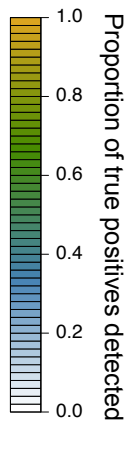

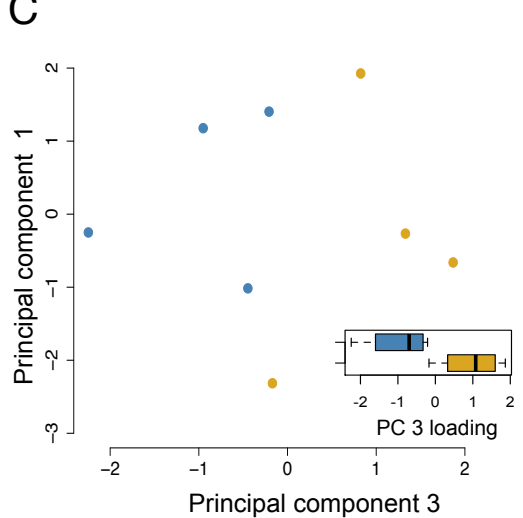

648

Figure 2. Estimates of effect sizes and their impact on the power of differential methylation analysis. (A) The maximum percent difference in mean DNA methylation levels between two sample groups ( $y$-axis), for selected percentiles of sites (x-axis, ranked from smallest to largest percent difference) in reanalyzed data sets (Table 1) with binary predictor variables. Mean differences are based on raw values, without correction for covariates that may be imperfectly balanced between sample groups (e.g., age of study subjects in the baboon data set). We focus on the largest percentiles here because those effects are most likely to be detected or of interest to most investigators. (B) Power to detect differentially methylated sites at a 5\% FDR in simulated RRBS datasets. Power increases as a function of the simulated sample size ( $y$-axis; note that sample size is plotted on a log scale) and the magnitude of the effect of interest on DNA methylation levels ( $x$-axis, represented as the proportion of variance in DNA methylation levels explained by the predictor variable). (C) In a small dataset $(n=8)$, site-by-site analyses are too underpowered to detect differential methylation between clonal raider ants in the reproductive versus brood care phases. However, principal components analysis clearly separates samples by caste (t-test for PC 3 , which explains $21.7 \%$ of the overall variance: $p=$ 0.022; inset compares PC 3 loadings for reproductive versus brood care samples). 
665

666

667

668

669

670

671

672

673

674

675

676
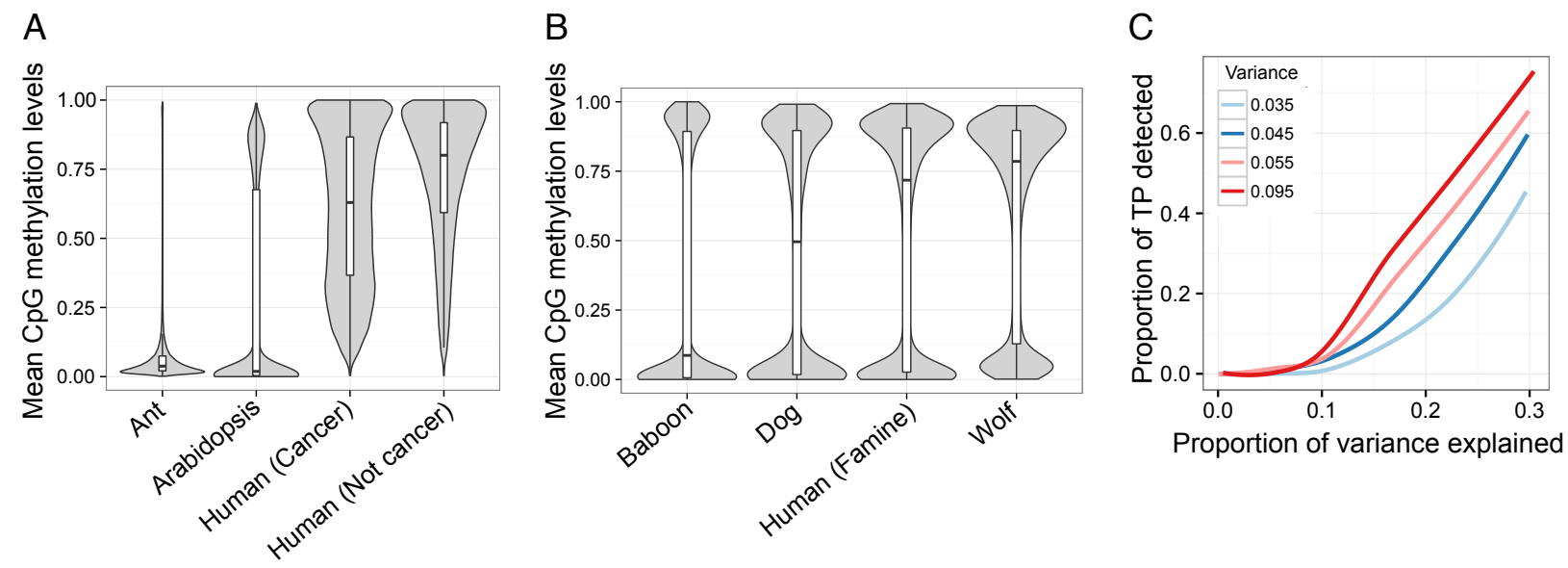

Figure 3. Properties of $\mathrm{CpG}$ methylation levels vary across data sets and influence power. For each (A) WGBS and (B) RRBS data set, we plotted the distribution of mean DNA methylation levels at each $\mathrm{CpG}$ site with a median coverage $>10 \mathrm{x}$ across all samples in the study. (C) Power to detect differentially methylated sites (at a 5\% FDR) in simulated RRBS datasets. The proportion of simulated true positives (TP) detected is plotted on the $y$-axis. Power increases as a function of the simulated effect size (represented as the proportion of variance explained; $x$ axis) and the variance in DNA methylation levels (colors). For all simulations, mean DNA methylation levels were held constant. The levels of variance in DNA methylation levels explored here $(0.035,0.045,0.055$, and 0.095$)$ represent common values observed in real bisulfite sequencing data sets (Figure S5). 